\title{
Erfassung von Aufmerksamkeitsdefiziten bei Patienten mit obstruktivem Schlafapnoesyndrom mit unterschiedlichen Fahrsimulationsprogrammen
}

\author{
A. Büttner \\ W. Randerath \\ K.-H. Rühle
}

\author{
Two Simulation Programs to Measure Continuous Attention \\ in Obstructive Sleep Apnea Syndrome
}

\section{Zusammenfassung}

Patienten mit obstruktivem Schlafapnoesyndrom (OSAS) leiden aufgrund neuropsychologischer Defizite an einer Minderung der Daueraufmerksamkeit. Zur Erfassung, Objektivierung sowie Therapieverlaufsbeobachtung werden verschiedene neuropsychologische Testverfahren eingesetzt. Unter der Annahme, dass die Fehlerrate bei Fahrsimulationen mittels Computer mit der realen Unfallhäufigkeit korrelieren, wurden verschiedene Fahrsimulationstests unterschiedlicher Komplexität entwickelt. Während Steer Clear (Findley u. Mitarb.) und der Fahrsimulator Carda (Randerath u. Mitarb.) nur sehr abstrakt eine Autofahrt mit plötzlich auftretenden Hindernissen simulieren, werden mit dem interaktiven Fahrsimulatortest Carsim - einem TrackingTest - komplexere Leistungen mit Steuertätigkeit erfasst. Um die Aussagekraft der beiden Verfahren abzuschätzen, wurden 105 OSAS-Patienten mittels beider Fahrsimulatoren Carda und Carsim bezüglich der Fehlerhäufigkeit überprüft. Unter Carda lag die Fehlerrate bei 10/105 Patienten (9,5\%) außerhalb des von uns verwandten Normbereiches (Mittelwert \pm zweifache Standardabweichung einer gesunden Population ohne schlafbezogene Atemstörungen). Bei Carsim war die Spurabweichungszeit bei 49/105 Patienten (46,7\%) außerhalb des Normbereiches. Die Häufigkeit der Abweichungen von der Norm war unter Carsim signifikant höher. Durch die zusätzliche Testung mit Carda erhöhte sich die Zahl der pathologischen Fälle von 46,7\% auf 51,4\%. Mit den beiden Tests werden unterschiedliche Komponenten neuropsychologischer Funktionsstörungen erfasst, Fahrsimulatortests mit Steuerungsaufgaben beschreiben im Vergleich zu Fahrsimulatoren mit lediglich Reaktionsprüfungen bei OSAS in einem höheren Prozentsatz neuropsychologische Defizite.

\section{Abstract}

Patients with obstructive sleep apnea syndrome suffer from reduced continuous attention due to neuropsychological deficits. Among other means, driving simulator programs are employed for registration and objectification as well as observation of the course of therapy. While Steer Clear by Findley et al. and the driving simulator Carda (Randerath et al.) represent pure continuous attention tests, the new driving simulator test Carsim, measures attention interactively and continuously. This way, more complex functions are recorded. We therefore investigated Carda and the program Carsim to study the various features of both methods. For this purpose, 105 OSAS patients were tested on both driving simulators concerning the mistake rate in Carda and the time of tracking deviations in Carsim. We defined the normal range by using the mean value \pm 2 standard deviations from our earlier publications in healthy persons without sleep disorders. With Carda the mistake rate exceeded in 10 of 105 patients (9.5\%) the normal range and with Carsim the frequency of tracking deviations exceeded in 49 of 105 patients (46.7\%) the normal range. The incidence of deviation from normal was significantly higher with Carsim testing. By addionally testing the number of pathological cases is with Carda increased from $46.7 \%$ to $51.4 \%$. The tests characterize different components of neuropsychological deficits. Driving simulators with tracking tasks describe neuropsychological deficits in comparison with those measuring only components of reaction in a higher percentage.

Institutsangaben

Klinik Ambrock, Klinik für Pneumologie, Allergologie und Schlafmedizin, Universität Witten-Herdecke, Hagen

Widmung

Herrn Prof. Dr. N. Konietzko zum 65. Geburtstag gewidmet

Korrespondenzadiresse

Dr. Antje Büttner · Klinik Ambrock · Klinik für Pneumologie · Ambrocker Weg 60 .

58091 Hagen ·E-mail: antja18B@yahoo.com

Eingang: 13. Mai 2003 · Nach Revision angenommen: 31. Juli 2003

Bibliografie

Pneumologie 2003; 57: 722-728 @ Georg Thieme Verlag Stuttgart · New York · ISSN 0934-8387 
Das obstruktive Schlafapnoesyndrom ist u.a. durch nächtliche Arousals und Schlaffragmentierung im Rahmen respiratorischer Ereignisse gekennzeichnet. Die aufgrund dessen tagsüber auftretende Müdigkeit beinhaltet auch eine Minderung der Daueraufmerksamkeit und der Vigilanz, insbesondere bei monotonen Tätigkeiten im Arbeitsleben [1 - 3,24, 25, 29, 30, 32,33,41].

Diese Beeinträchtigung führt wiederum zu einer geminderten Leistungsfähigkeit und beeinflusst außerdem die Fähigkeit der Betroffenen, Kraftfahrzeuge zu führen sowie die Erwerbstätigkeit, speziell bei Berufen mit erhöhtem Gefahrenpotenzial [10-12,14-18,22,31,34].

Eine Feststellung des Wachheitsgrades ist anhand von Selbsteinschätzungen mittels Fragebogen (Epworth Sleepiness Scale ESS $[27,28]$, Stanford Sleepiness Scale SSS [26]) oder durch standardisierte Testverfahren (Multiple Sleep Latency Test MSLT, Maintainance of Wakefulness MWT $[43,44])$ möglich.

Da neuropsychologische Defizite bei Schlafapnoesyndromen häufig auftreten, wurden verschiedene Testverfahren zur Beschreibung des Schlafapnoesyndroms als auch Verlaufsbeobachtung unter nCPAP-Therapie eingesetzt. Da nachgewiesen werden konnte, dass sowohl die Unfallrate als auch Beinaheunfälle bei OSAS erhöht sind und unter nCPAP-Therapie reduziert werden können [31], bietet es sich an, Fahrsimulationsprogramme zu verwenden.

Unter der Annahme, dass die Fehlerrate bei Fahrsimulationen mittels Computer mit der realen Unfallhäufigkeit korrelieren, wurden verschiedene Fahrsimulationstests unterschiedlicher Komplexität eingesetzt. Diese erfassen einerseits die Daueraufmerksamkeit global und spiegeln andererseits auch essenzielle Aspekte (tracking; visual search) der komplexen Leistung des Autofahrens bzw. der Fahrtüchtigkeit wider [5-8,13,14, $24,39,40]$. So wiesen u.a. Moskowitz und Robinson einen Zusammenhang zwischen Aufmerksamkeit, Alkohol am Steuer und Fahrsimulatortestung (tracking; visual search) im Labor nach $[35,36]$.

Die entscheidenden Komponenten sind hierbei das Steuern (tracking) sowie das Absuchen der Umgebung nach relevanten Reizen (visual search). Auf dieser Grundlage entwickelten George u. Mitarb. ihr Fahrsimulationsprogramm, das auf dem Test von Moscowitz u. Mitarb. zur geteilten Aufmerksamkeit beruht [19-21,23].

Auf dieser theoretischen Konzeption basiert auch der neu entwickelte Fahrsimulator (Carsim).

Das heute häufig verwendete Modell zur Aufmerksamkeit stammt von Posner und Petersen (1990; [38]). Sie verbanden die Konstrukte der Kapazitätsbegrenzung, der Verarbeitungskontrolle und der Aufmerksamkeitsselektivität miteinander und unterscheiden zwischen einem Orientierungs-, einem Selektivitäts- und einem Vigilanzsystem [37,38].

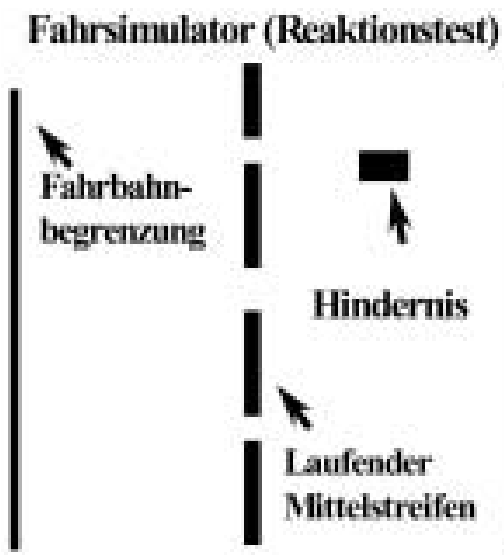

Abb. 1 Fahrsimulator Carda nach Gerdesmeyer u. Mitarb. 1997, Randerath u. Mitarb. 1997, 1998. Bei dem Vigilanztest Carda wird das Bild einer Straße mit Randbegrenzung und Mittelstreifen vor einem schwarzen Hintergrund projiziert. Auf diesem leuchten kurzzeitig zeitlich und örtlich zufällig Hindernisse in Rechteckform auf, wobei pro 10-Minuten-Abschnitt immer 100 Ereignisse präsentiert werden. Der Patient muss innerhalb einer Sekunde anhand der Leertaste auf sie reagieren.

Im Zusammenhang mit unserer Studie ist besonders das Vigilanzsystem von Interesse. Laut Posner und Rafal (1987; [37]) sowie Posners und Petersens (1990; [38]) Theorie dient es besonders der Fokussierung der Aufmerksamkeit und ist speziell in monotonen und längerwährenden Situationen von Bedeutung $[37,38]$.

Die Konzeption des Fahrsimulators Carda basiert auf der theoretischen Grundlage des Vigilanzkonzeptes von Rützel und Brickenkamp [4,42]. Bei diesem Daueraufmerksamkeitstest sind die Bedingungen eines eintönigen Reizhintergrundes sowohl zeitlich und örtlich nicht voraussagbarer Ereignisse integriert ([24,39,40]; Abb.1). Auch der neu entwickelte Fahrsimulator Carsim basiert auf diesem Prinzip, neben den Reaktionszeiten bei Auftauchen von Verbotsschildern werden zusätzlich die Spurabweichungen erfasst ([5-8]; Abb.2). Während Carda jedoch aufgrund der Testkonstruktion als reiner Daueraufmerksamkeitstest mit Erfassung fehlender Reaktionen entwickelt wurde, stellt Carsim eine interaktive Fahrsimulation dar (siehe Tab.1).

Tab. 1 Konzeption und Eigenschaften der beiden Simulationsprogramme

\begin{tabular}{lll} 
& Carda & Carsim \\
\hline Monotonie & $(+)$ & +++ \\
\hline Kontinuität & - & ++ \\
Interaktivität & - & +++ \\
\hline Bedienbarkeit & +++ & ++ \\
\hline
\end{tabular}

$+=$ positiv; - = negativ

Es ergaben sich deshalb die folgenden Fragestellungen:

- Wie hoch ist der prozentuale Anteil der Patienten mit pathologischen Fahrsimulator-Testergebnissen?

- Bestehen Zusammenhänge zwischen der mit der ESS erfassten Schläfrigkeit der Patienten und den Ergebnissen der beiden Fahrsimulatoren? 


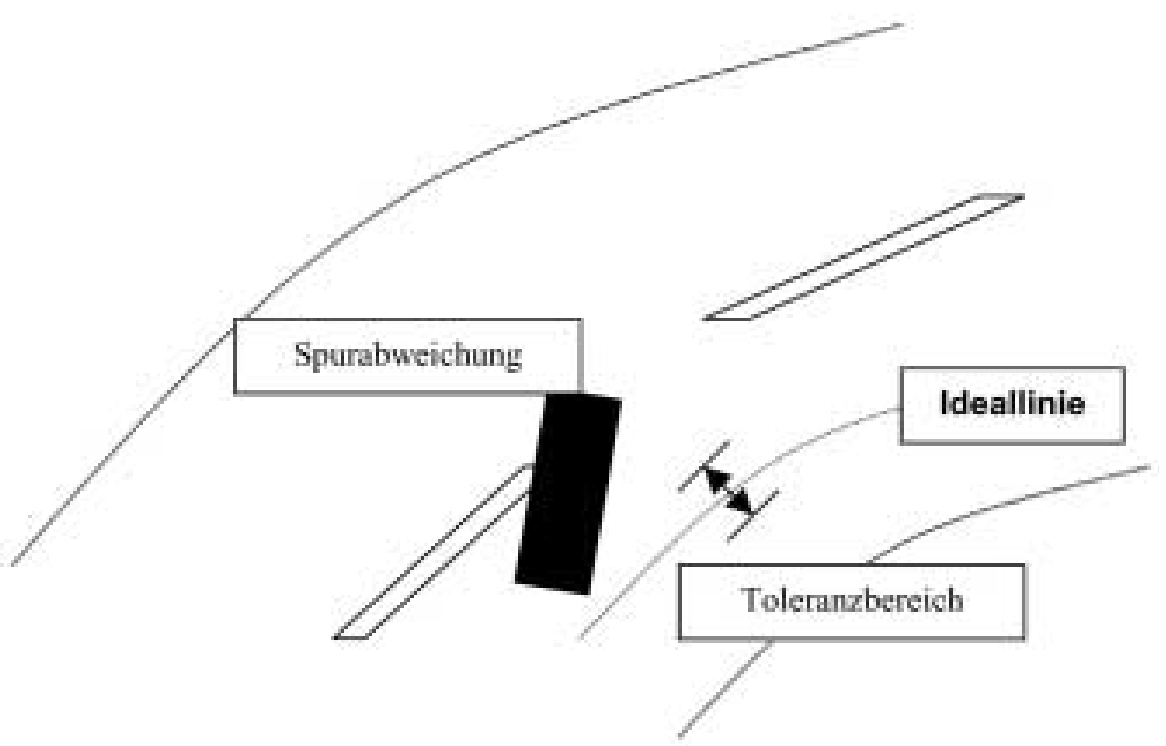

Abb. 2 Fahrsimulator Carsim nach Büttner u. Mitarb. 1999, 2000. Bei Carsim wird das Bild einer Straße mit Randbegrenzung und Mittelstreifen dargestellt. Der Patient bzw. Proband hat nun z. B. die Aufgabe, mithilfe eines Lenkrades auf seiner Fahrbahn die Idealspur zu halten (tracking) und anhand zweier Knöpfe, die sich an der Lenkradkonsole befinden, auf die auftauchenden Hindernisse (Verkehrszeichen) zu reagieren (visual search).

Methodik

\section{Messgeräte}

\section{Erfassung der Daueraufmerksamkeit: \\ Carda}

Allgemeines: Carda ist ein computergestützter Reaktionstest zur Erfassung der Vigilanz, der mittels eines menügesteuerten Turbo-Pascal-Programms auf einem handelsüblichen PC betriebenen werden kann.

Durchführung: Bei Carda wird das Bild einer Straße mit Randbegrenzung und Mittelstreifen vor einem schwarzen Hintergrund projiziert. Auf diesem leuchten Hindernisse in Rechteckform auf, die jeweils $20 \mathrm{~ms}$ sichtbar sind. Dies erfolgt zeitlich und örtlich zufällig, wobei pro 10-Minuten-Abschnitt immer 100 Ereignisse präsentiert werden. Der Patient muss innerhalb einer Sekunde anhand der Leertaste auf sie reagieren.

Die Testzeit beträgt $30 \mathrm{~min}$, eine Übungsphase ist aufgrund der einfachen Testdurchführung nicht erforderlich. In der klinischen Routine erfolgt keine Überwachung des Patienten.

Auswertung: Die Berechnung der Fehler erfolgt in Relation zu den dargebotenen Ereignissen, die der unbegründeten Reaktionen in absoluten Zahlen ([24,39,40]; Abb.1). Unterbliebene Reaktionen werden als Fehler registriert, verspätete als unbegründete Reaktionen. Ausgewertet werden nur unterbliebene Reaktionen.

Objektivität: Testvorgabe, Registrierung und Auswertung aller Messwerte erfolgten automatisch. Die Objektivität ist somit per se gegeben, da sowohl eine Beeinflussung der Versuchspersonen untereinander als auch ein Versuchsleitereinfluss aufgrund der Testkonzeption ausgeschlossen sind.

Reliabilität: Reliabilitätsprüfungen sind nicht bekannt.

Validität: Validitätsuntersuchungen existieren nicht. Jedoch gewährleistet Carda eine hohe Augenscheinvalidität. So sind seine Ergebnisse eindeutig interpretierbar, plausible Alternativerklä- rungen aufgrund nicht kontrollierter Störvariablen (Kontrolle der Störvariablen) existieren nicht (= interne Validität). Die Ergebnisse sind über die Besonderheit der Untersuchungssituation sowie die untersuchten Personen hinaus generalisierbar, da keine extreme Unnatürlichkeit in der Untersuchungssituation vorliegt und die Stichprobe zusätzlich repräsentativ ist (= externe Validität).

Standardisierung: Carda ist so konzipiert, dass Probanden und Patienten unter denselben Untersuchungsbedingungen getestet werden, wobei die Erfassung der Vigilanz das einzige variable Merkmal darstellt.

Normierung: Die mittlere Fehlerrate lag bei 5,75\% $\pm 11,3$ (Normalkollektiv: $n=63$ ). Es konnte keinerlei Einfluss von Alter, Geschlecht und BMI gefunden werden [40].

Ökonomie: Aufgrund des geringen Zeitaufwandes, der computergestützten Durchführung und der automatischen Testauswertung ist Carda ein ökonomischer Reaktionstest.

Beurteilung: Bei Carda handelt es sich um ein ökonomisches Testverfahren zur Erfassung der Vigilanz. Wünschenswert wären Angaben zur Reliabilität und zur Konstruktvalidität.

\section{Carsim}

Allgemeines: Bei Carsim handelt es sich ein um menügesteuertes Turbo-Pascal-Programm zur Erfassung der Daueraufmerksamkeit, das auf einem handelsüblichen PC betriebenen werden kann.

Durchführung: Bei Carsim wird das Bild einer Straße mit Randbegrenzung und Mittelstreifen polychrom simuliert. An der rechten Straßenseite können Hindernisse in Form von Durchfahrtsverbotsschildern präsentiert werden, die jeweils nur kurz sichtbar sind (z. B. für $200 \mathrm{~ms}$ ). Ihr Erscheinen erfolgt zeitlich zufällig, wobei in einem 5-Minuten-Abschnitt eine fixe Anzahl von Ereignissen eingestellt werden kann. Der Patient hat nun die Aufgabe, mithilfe eines Lenkrades auf seiner Fahrbahn die Idealspur zu halten (tracking) und anhand zweier Knöpfe (beide gleiche Funktion), die sich an der Lenkradkonsole befinden, auf die auftauchenden Hindernisse zu reagieren (visual search). 
Die Testzeit beträgt 30 min mit einer zuvor 5-minütigen Übungsphase. Bei den klinischen Untersuchungen erfolgt eine Kameraüberwachung des Patienten.

Auswertung: Abhängig von den Lenkradbewegungen wird die Position des Fahrzeugs auf der Straße jeweils neu berechnet und online visualisiert. Das Programm registriert die Abweichungszeit von der Ideallinie (Toleranz) und von der Spur (Spurabweichungszeit) sowie die richtigen, die fehlenden, die unbegründeten Reaktionen und die Reaktionszeit ([5-8]; Abb. 2).

Unter Toleranz- bzw. Spurüberschreitung versteht man die Anzahl der Tracking-Fehler, die absolut gesehen, die im Test vorgegebene Toleranz bzw. Spurbreite überschreiten. Durch Umrechnung der Anzahl der Pixel erhält man die Zeit in Sekunden, die außerhalb des Toleranzbereiches bzw. außerhalb der Fahrbahn gefahren wurde.

Objektivität: Testvorgabe, Registrierung und Auswertung aller Messwerte erfolgen automatisch. Die Objektivität ist somit per se gegeben, da sowohl eine Beeinflussung der Versuchspersonen untereinander als auch ein Einfluss des Versuchsleiters aufgrund der Testkonzeption ausgeschlossen sind.

Reliabilität: Die Überprüfung der Reliabilität mithilfe des Cronbach- $\alpha$ betrug für die tracking-Komponente $r=0,9785$, für das Visual Search $r=0,9666$ und für die Reaktionszeit $r=0,8943$, die der Retest-Reliabilität (nach 3 Tagen) für die Tracking-Komponente $r_{t t}=0,9855$, für das Visual Search $r_{t t}=0,9447$ und für die Reaktionszeit $r_{\mathrm{tt}}=0,9211$.

Validität: Außerdem gewährleistet carsim eine hohe Validität. So sind seine Ergebnisse eindeutig interpretierbar, plausible Alternativerklärungen aufgrund nicht kontrollierter Störvariablen (Kontrolle der Störvariablen) existieren nicht (= interne Validität). Die Ergebnisse sind über die Besonderheit der Untersuchungssituation sowie die untersuchten Personen hinaus generalisierbar, da keine extreme Unnatürlichkeit in der Untersuchungssituation vorliegt und die Stichprobe zusätzlich repräsentativ ist ( $=$ externe Validität). Die Konstruktvalidität des Tests liegt bei $R=0,125 ; p=0,026$.

Standardisierung: Carsim ist so konzepiert, dass Probanden und Patienten unter denselben Untersuchungsbedingungen (Tab. 2) getestet werden, wobei die Erfassung der Vigilanz das einzige variable Merkmal darstellt.

Normierung: Der mittlere Fehler der Spurabweichungszeit lag in der Eichstichprobe bei 2,3 $\pm 4,5$ s, der Grenzwert der Spurabweichungen der Gesunden in einem 95\%-Konfidenzintervall bei $<13,2$ s.

98\% der Gesunden erreichten Werte zwischen 0 bis 150 Abweichungen von der Spur.

Der mittlere Fehler der Toleranzabweichungszeit lag bei den Gesunden bei $96,0 \pm 177,0$ s, der Grenzwert der Toleranzabweichungen der Eichstichprobe in einem 95\%-Konfidenzintervall bei $<450,4$ s.
Tab. 2 Standardeinstellung von Carsim

\begin{tabular}{|ll}
\hline Testparameter & Standardeinstellung \\
\hline Testlänge & $30 \mathrm{~min}$ (6 Sektionen à $5 \mathrm{~min}$ ) \\
\hline Übungsphase & $5 \mathrm{~min}$ \\
\hline Geschwindigkeit & $100 \mathrm{~km} / \mathrm{h}$ \\
\hline Anzahl der Kurven & 1 je Sektion \\
\hline Anzahl der Hindernisse & 3 je Sektion \\
\hline Sichtbarkeit der Hindernisse & $200 \mathrm{~ms}$ \\
\hline
\end{tabular}

99\% der Gesunden erreichten Werte zwischen 0 bis $<10000 \mathrm{Ab}-$ weichungen von der Toleranz $[5,8]$.

Ökonomie: Carsim ist aufgrund des geringen Zeitaufwandes, der computergestützten Durchführung und der automatischen Testauswertung ein sehr ökonomischer Daueraufmerksamkeitstest dar.

Beurteilung: Bei Carsim handelt es sich um ein ökonomisches Testverfahren zur Erfassung der Daueraufmerksamkeit. Wünschenswert wären Untersuchungen zur Vergleichbarkeit mit anderen Daueraufmerksamkeitstests.

\section{Erfassung der subjektien Schlafqualität: Epworth Sleepiness Scale (ESS)}

Die Epworth Sleepiness Scale erhebt anhand einer Selbstbeurteilung die Wahrscheinlichkeit des Einnickens in bestimmten Situationen während des Tages. Die durchschnittliche Bearbeitungszeit beträgt ein bis zwei Minuten.

Die ESS umfasst acht Fragen bzw. Items zur Tagesmüdigkeit (bspw. im Sitzen, beim Fernsehen oder beim Autofahren). Itemund Faktoranalysen ergaben bei der ESS eine sehr gute Interne Konsistenz (Cronbach- $\alpha=, 88$ ) und eine hohe Retest-Reliabilität nach fünf Monaten bei Normalpersonen $\left(r_{t t}=, 822 ; n=87\right.$; $\mathrm{p}<0,001)[27,28]$.

\section{Fragebogen}

Mit einem speziellen Fragebogen können Alter, Geschlecht, Schulbildung, der Beruf, die subjektiven Beeinträchtigungen bezüglich Sehfähigkeit und Motorik (u.a. Störungen der Bewegungskoordination z.B. Zittern, Schwäche oder Lähmungserscheinungen der oberen Extremitäten) sowie die Dauer der Fahrpraxis erfasst werden.

Die subjektiven schlafbezogenen Angaben beschreiben eventuelle Erkrankungssymptome sowie deren eingeschätzte Relevanz (z. B. bezüglich körperlicher und geistiger Leistungsfähigkeit, Tagesmüdigkeit und Unfallhäufigkeit) $[5,8]$.

Auswertet wurden in der Untersuchung nur die demografischen Angaben.

\section{Studiendesign}

Bei den Patienten wurde zunächst eine komplette Polysomnographie nach den Kriterien der DGSM zur Diagnosestellung und/ 
oder-sicherung durchgeführt, außerdem eine orientierende psychiatrische/neurologische Untersuchung.

Die Messung der Fahrsimulatortestungen erfolgte jeweils nachmittags und fand in einem unbeleuchteten, abgedunkelten und schallisolierten Raum statt. Die Testinstruktion war standardisiert und wurde immer durch denselben Versuchsleiter gegeben.

Die Studienteilnehmer absolvierten randomisiert cross-over im Abstand von einem Tag sowohl den Vigilanztest Carda als auch den Fahrsimulator Carsim über eine Zeitdauer von 30 Minuten (vor Therapieeinleitung).

Ausgewertet wurden nur

- bei Carda die Anzahl der fehlerhaften Reaktionen

- bei Carsim die Spurabweichungszeit.

\section{Einschlusskriterien}

Die Teilnahme an der Studie war freiwillig; bei allen Patienten musste eine Einwilligungserklärung (informed consent) vorliegen.

Die Diagnose obstruktives Schlafapnoesyndrom musste nach ICD-9 vorliegen und durch eine Polysomnographie nachgewiesen/bewiesen sein. Der AHI (Apnoe-Hypopnoe-Index) musste einen Wert größer als 10/h aufweisen.

Die Studienteilnehmer durften 4-6 Stunden vor der Untersuchung weder Alkohol, Nikotin noch koffeinhaltige Getränke zu sich genommen haben. Sie sollten nicht unter einem Hungeroder Durstgefühl leiden. Außerdem durften sie keine aufmerksamkeitsbeeinträchtigenden Medikamente eingenommen haben.

\section{Ausschlusskriterien}

Die Studienteilnehmer sollten nicht älter als 70 Jahre alt sein. Sie durften nicht unter einem nicht OSAS-bedingtem Schnarchen leiden, keine andersartigen Atemwegserkrankungen sowie keine psychiatrischen/neurologischen Erkrankungen aufweisen. Die Probanden sollten außerdem unter keinerlei anderen bekannten Schlafstörungen und/oder -problemen leiden.

\section{Statistik}

Zur Berechnung der Ergebnisse wurde das Programm Statistical Package for Social Sciences (SPSS) verwendet. Die Ergebnisse wurden deskriptiv durch den Mittelwert \pm Standardabweichung (SD) beschrieben. Die zu testenden Variablen wurden mit dem $t$-Test für abhängige Variablen verglichen.

Der Vergleich der beiden Fahrsimulatoren bez. pathologischer Fälle wurde mit dem nichtparametrischen Test McNemar durchgeführt.

Eine Signifikanz wurde bei einem $\mathrm{p}<0,05$ angenommen.

\section{Patientengut}

Nachdem Patienten mit Störungen der Sehfähigkeit und der Bewegungskoordination z.B. Zittern, Schwäche oder Lähmungserscheinungen der oberen Extremitäten von der Untersuchung ausgeschlossen waren, absolvierten 137 konsekutive OSAS-Pa- tienten Carsim und Carda, 29 Frauen und 108 Männer. Von diesen wurden weitere 32 Patienten (12 Männer und 20 Frauen) ausgeschlossen, da sie den Testablauf nicht ordnungsgemäß absolvierten. Ausschlüsse wurden bei Patienten vorgenommen, die eins der beiden Testverfahren nicht ordnungsgemäß ausführten oder die Testung abbrachen (Angst-, Panikattacken wegen Dunkelheit oder geschlossener Tür; körperliches Unwohlsein) bzw. die Probleme bei der Durchführung des Tracking-Tests hatten.

Der Altersmittelwert der restlichen Studienteilnehmer $(n=105)$ lag bei $53,9 \pm 11,8$ Jahren. Der Apnoe-Hypopnoe-Index (AHI) betrug $32,5 \pm 21,3 / \mathrm{h}$, die tiefste Sauerstoffsättigung lag bei $82,6 \pm$ $7,6 \%$.

Ergebnisse

\section{Epworth-Score und Unfallhäufigkeit}

58 Patienten (55,2\%) wiesen einen Epworth-Score von $\geq 9$ (Normalbereich <9) auf.

76 Personen (72,4\% der OSAS-Patienten) gaben an, keinen Unfall in den letzten fünf Jahren gehabt zu haben, 27 (25,7\%) gaben weniger als fünf Unfälle (21 Personen einen Unfall, 4 zwei Unfälle, 2 drei), 2 (1,9\%) mehr als fünf Unfälle im selben Zeitraum an.

\section{Carda vs Carsim}

Unter Carda lag die Fehlerrate bei 10/105 Patienten (9,5\%) außerhalb des Normbereiches. Bei Carsim war die Spurabweichungszeit bei 49/105 Patienten (46,7\%) außerhalb des Normbereiches (Abb.3). Die Häufigkeit der Abweichungen von der Norm war unter Carsim signifikant höher $(\mathrm{p}<0,001)$.

Durch die zusätzliche Testung mit Carda erhöhte sich die Zahl der pathologischen Fälle von 46,7\% auf 51,4\%.

Eine signifikante Korrelation zwischen den Fehlerraten der Fahrsimulatoren und dem Epworth-Score konnte nicht gefunden werden.

Der Korrelationskoeffizient $(\mathrm{n}=105)$ der Beziehung zwischen der Tracking-Variablen (Spurabweichungszeit) des Fahrsimulators Carsim und dem Epworth-Score (ESS) lag bei $r=-0,011$ $(\mathrm{p}=0,896$; ns.), der korrelative Zusammenhang $(\mathrm{n}=105)$ zwischen der Fehlerrate (\%) von Carda und dem Epworth-Score (ESS) bei $r=0,111(p=0,209$; $n s$. .

\section{Diskussion}

Der Fahrsimulator Carda, ähnlich wie der von Findley entwickelte Test, entspricht nicht den Anforderungen, die an einen echten Tracking-Test gestellt werden. Es handelt sich vielmehr um einen Reaktionstest, der die Aufmerksamkeit und die Vigilanz beschreibt.

Krieger u. Mitarb. konnten bereits mittels Fragebogen nachweisen, dass die Unfallrate bei OSAS-Patienten häufig durch Schläfrigkeit ausgelöst wurde und dass sowohl die Unfallrate als auch Beinahunfälle unter nCPAP-Therapie reduziert werden konnten 


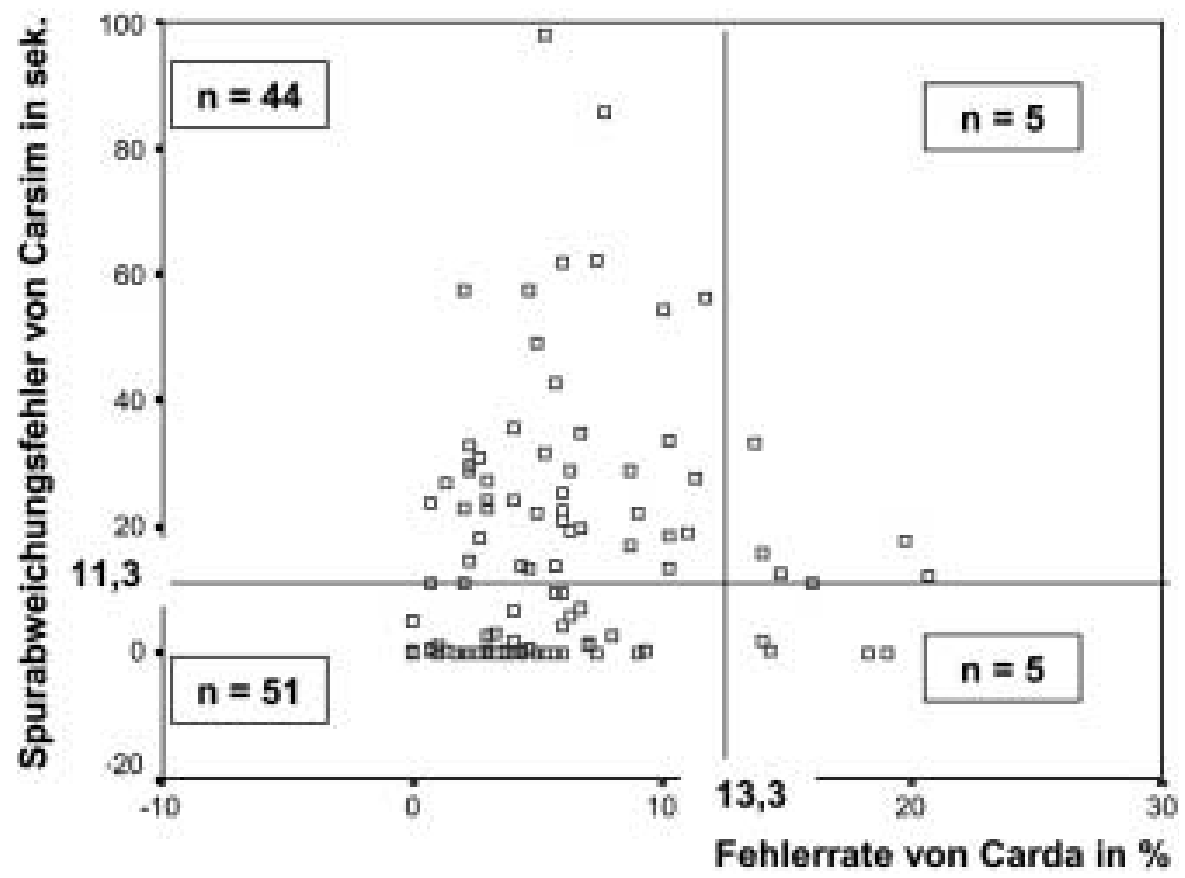

Abb. 3 Spurabweichung Carsim (aV) und Vigilanztest Carda (uV). Scatter-Diagramm des Vergleichs der Fehlerrate von Carda und der Spurabweichungszeit in der Untersuchung mit dem interaktiven Fahrsimulator Carsim aller $105 \mathrm{~Pa}$ tienten. Eingezeichnet sind die Grenzen des Normalbereichs für Carda $<13,3$ \% Fehler und Carsim $<11,3$ s Spurabweichungszeit.

[31]. Ein Test zur Abschätzung des Unfallrisikos wäre deshalb hilfreich.

Findley fand eine Korrelation zwischen der Anzahl der Unfälle und der Fehlerrate beim Fahrsimulatortest Steer Clear anhand von Daten der Unfallbehörde Virginias/USA. Weiterhin wies er einen gewissen Zusammenhang zwischen Unfallhäufigkeit und Schlafapnoesyndrom sowie eine Abhängigkeit vom Schweregrad der Erkrankung nach [13,16 - 18]. Tests dieser Art sollten unserer Ansicht nach nur mit aller Vorsicht bei der Frage der Fahrtüchtigkeit verwandt werden, da sie nur wenige Aspekte erfassen.

Aufgrund der einfachen Konstruktion bieten Steer Clear und Car$d a$ aber auch einige Vorteile, da der technische Aufwand relativ gering ist und selbst eingeschränkte Patienten die Aufgabe gut verstehen. Allerdings kann mit den Tests nur bei deutlich erhöhter Fehlerquote das Ansprechen auf CPAP überprüft und der Verlauf kontrolliert werden.

Der Schweregrad der Schläfrigkeit, beurteilt durch die ESS, korrelierte nicht mit den Ergebnissen der Fahrsimulatoren. Schläfrigkeit beschreibt den Grad der Wachheit und wird durch zentralnervöse Aktivierung beeinflusst [44]. Durch die Testsituation wird bei mäßiger Einschränkung (ESS < 13) die Schläfrigkeit häufig kompensiert - bei unseren Patienten lag der ESS-Score im Mittel bei 11,0 -, so dass die Fehlerzahl bzw. die Spurüberschreitung keine relevante Abhängigkeit zeigten. Allerdings wurde bei ausprägter Schläfrigkeit von OSAS-Patienten mit einem ESSScore > 13 mit Carda eine höhere Fehlerzahl erreicht [40].

Unter der Testung mit Carsim lag die Anzahl der Patienten, die eine sichere pathologische Abweichung aufwiesen deutlich höher.

Die komplexere Aufgabe der interaktiven Fahrsimulation erfasst Patienten mit eingeschränkter Leistungsfähigkeit bezüglich geteilter Aufmerksamkeit und interaktivem Handeln. Dies konnte an Personen, deren Fahrleistung nach Alkoholgabe mit einem Fahrsimulator überprüft wurde, nachgewiesen werden. Bei OSAS war die Leistung ähnlich eingeschränkt wie bei einem Promille-Gehalt von $95 \pm 25 \mathrm{mg} / \mathrm{dl}$ Alkohol im Blut [19].

Untersuchungen, die Laborergebnisse von Tracking-Tests mit der tatsächlichen Unfallhäufigkeit korrelieren, fehlen noch. Es wäre deshalb wünschenswert, objektive Daten der Straßenverkehrsbehörden zu erhalten, um evtl. Risikopatienten mit diesem sensitiven Instrument besser charakterisieren zu können. Ein Tracking-Fahrsimulator besitzt zwar einen höheren Realitätscharakter, da er die Aufgabe, d.h. die Fahrsituation besser als ein Reaktionstest abbildet [23]. Dennoch ist auch hier Skepsis an seiner Aussagekraft angebracht, da Fahrtüchtigkeit von sehr vielen Faktoren (z. B. von verantwortungsvollem Handeln) abhängt, die mit Simulationstests allein nicht erfasst werden können.

Ein Fahrsimulatortest sollte daher immer nur als eine von mehreren Komponenten bei der komplexen Beurteilung der Fahrtüchtigkeit verwandt werden.

Der von uns konzipierte interaktive Fahrsimulatortest kann auBerdem für weitere Fragestellungen eingesetzt werden: Bei Patienten mit OSAS lassen sich durch unterschiedliche Therapiemodalitäten mehrere Teilkomponenten der Aufmerksamkeit (u. a. selektive und geteilte Aufmerksamkeit, Daueraufmerksamkeit, Verarbeitungsgeschwindigkeit) verbessern [6,9]. Ein interaktiver Fahrsimulator, der mehrere dieser Veränderungen reflektiert, sollte ein geeigneteres Instrument darstellen, da TrackingAufgaben im Vergleich zu Reaktionstests (Carda) mehr Komponenten der eingeschränkten Leistungsfähigkeit abbilden.

Wir konnten in dieser Untersuchung nachweisen, dass ein interaktiver Fahrsimulator (z.B. Carsim) die Störung von Patienten mit OSAS sensitiver beschreibt. Er wird von uns daher gezielt in klinischen Studien für die Beurteilung von Therapieeffekten zur 
Aufmerksamkeitssteigerung (z. B. unter CPAP oder Theophyllin; $[6,9]$ ) eingesetzt. Aufgrund der einfachen Handhabung wird aber auch Carda in der klinischen Routine weiterhin eine geeignete Methode bleiben, um neuropsychologische Störungen zu erfassen und Therapieeffekte nachzuweisen.

\section{Literatur}

${ }^{1}$ American Thoracic Society (ATS). Sleep apnea, sleepiness and driving risk. Am J Respir Crit Care Med 1994; 150: 1464-1473

2 Bédard MA, Montplaisir J, Richer F et al. Nocturnal hypoxemia as a determinant of vigilance impairment in sleep apnea syndrome. Chest 1991; 100: 367-370

${ }^{3}$ Bradley TD, Rutherford R, Grossman RF et al. Role of daytime hypoxemia in the pathogenesis of right heart failure in the obstructive sleep apnea syndrome. Am Rev Respir Dis 1985; 131: 835 - 839

${ }^{4}$ Brickenkamp R, Karl GA. Geräte zur Messung von Aufmerksamkeit, Konzentration und Vigilanz. In: Brickenkamp R (Hrsg.): Handbuch apparativer Verfahren in der Psychologie. Göttingen: 1986: 195-203

${ }^{5}$ Büttner A, Randerath W, Rühle KH. Fahrsimulatorvalidierung. Somnologie 1999; 3 (Suppl. 1): 9-10

${ }^{6}$ Büttner A, Randerath W, Rühle KH. Therapieverlaufskontrolle der Daueraufmerksamkeit anhand eines neuen Fahrsimulatortests bei OSAS-Patienten. Somnologie 1999; 3 (Suppl. 1): 10

7 Büttner A, Randerath W, Rühle KH. Der Fahrsimulatortest „Carsim“ zur Erfassung der Vigilanzminderung von SAS-Patienten. Einfluss verschiedener Faktoren auf die Normwerte. Pneumologie 2000; 54: $338-344$

${ }^{8}$ Büttner A, Randerath W, Rühle KH. Normwerte und Gütekriterien eines interaktiven Fahrsimulators („Carsim“). Somnologie 2000; 4: $129-136$

${ }^{9}$ Büttner A, Rühle KH. The Therapeutic Effect of Theophylline on Sustained Attention in Patients with Obstructive Sleep Apnea. Somnologie 2003; 7 (1): $23-27$

${ }^{10}$ Cassel W, Ploch T. Sleep apnea accidents: Health risk for healthy people? In: Peter JH, Penzel T, Podszus T, et al. (Hrsg.): Sleep and Health Risk. Berlin, Heidelberg, New York: 1991: 279-285

${ }^{11}$ Cassel W, Ploch T, Becker $C$ et al. Risk of traffic accidents in patients with sleep-disordered breathing: reduction with nasal CPAP. Eur Respir J 1996; 9: 2606-2611

12 Findley LJ, Unverzagt ME, Suratt PM. Automobile accidents involving patients with obstructive sleep apnea. Am Rev Respir Dis 1988; 138: $337-340$

${ }^{13}$ Findley LJ, Fabrizio MJ, Knight $\mathrm{H}$ et al. Driving simulator performance in patients with sleep apnea. Am Rev Respir Dis 1989; 140: 529-530

${ }^{14}$ Findley LJ. Automobile driving in sleep apnea. In: Sleep and Respiration. Wiley-Liss, Inc., 1990: 337-345

${ }^{15}$ Findley LJ, Weiss WJ, Jabour ER. Drivers with untreated sleep apnea: a cause of death and serious injury. Arch Intern Med 1991; 151: $1451-1452$

${ }^{16}$ Findley LJ, Unverzagt M, Guchu R et al. Vigilance and automobile accidents in patients with sleep apnea or narcolepsy. Chest 1995; 108: $619-624$

${ }^{17}$ Findley LJ, Suratt PM, Dinges DF. Time-on-task decrements in „steer clear" performance of patients with sleep apnea and narcolepsy. Sleep 1999; 22: 804-809

${ }^{18}$ Findley LJ, Smith C, Hooper J et al. Treatment with Nasal CPAP Decreases Automobile Accidents in Patients with Sleep Apnea. Am J Respir Crit Care Med 2000; 161: 857-859

${ }^{19}$ George CFP, Boudreau AC, Smiley A. Simulated driving performance in patients with obstructive sleep apnea. Am J Respir Crit Care Med 1996; 154: $175-181$

${ }^{20}$ George CFP, Boudreau AC, Smiley A. Comparison of simulated driving perfomance in narcolepsy and sleep apnea patients. Sleep 1996; 19: $711-717$
${ }^{21}$ George CFP, Boudreau AC, Smiley A. Effects of nasal CPAP on simulated driving performance in patients with obstructive sleep apnea. Thorax 1997; 52: $648-653$

22 George CFP, Smiley A. Sleep apnea and automobile crashes. Sleep 1999; 22: $790-795$

${ }^{23}$ George CFP. Vigilance impairment: assessment by driving simulators. Sleep 2000; 23 (4): 115-118

${ }^{24}$ Gerdesmeyer C, Randerath W, Rühle KH. Zeitliche Abhängigkeit der Fehlerzahl bei Messung der Daueraufmerksamkeit mittels Fahrsimulator vor und nach nCPAP-Therapie bei Schlafapnoesyndrom. Somnologie 1997; 1 : $165-170$

${ }^{25} \mathrm{He}$ J, Kryger MH, Zorick FJ et al. Mortality and apnea index in obstructive sleep apnea. Experience in 385-male patients. Chest 1988; 94: $9-14$

${ }^{26}$ Hoddes E, Zarcone V, Smythe $\mathrm{H}$ et al. Quantification of sleepiness: a new approach. Psychophysiology 1973; 10: $431-436$

${ }^{27}$ Johns MW. A new method of measuring daytime sleepiness: the Epworth sleepiness scale. Sleep 1991; 14: 540-545

28 Johns MW et al. Daytime Sleepiness, Snoring and Obstructive Sleep Apnea, The Epworth Sleepiness Scale. Chest 1993; 103: 30-36

${ }^{29}$ Kribbs NB, Getsy JE, Dinges DF. Investigation and management of daytime sleepiness in sleep apnea. In: Saunder NA, Sullivan CE (Hrsg.). Sleeping and Breathing 2. New York: 1993: 575-604

${ }^{30}$ Kribbs NB, Pack AL, Kline LR et al. Effects of one night without nasal CPAP treatment on sleep and sleepiness in patients with obstructive sleep apnea. Am Rev Respir Dis 1993; 147: 1162 - 1168

${ }^{31}$ Krieger J, Meslier N, Lebrun T et al. Accidents in obstructive sleep apnea patients treated with nasal continuous positive airway pressure. A prospective study. Chest 1997; 112: 1561 - 1566

32 Lamphere J, Roehrs T, Wittig R et al. Recovery of alertness after CPAP in apnea. Chest 1989; 96: 1364-1367

${ }^{33}$ Martin SE, Engleman HM, Deary IJ et al. The effect of sleep fragmentation on daytime function. Am J Respir Crit Care Med 1996; 153: $1328-1332$

${ }^{34}$ Mitler M, Carskadon M, Czeiler C et al. Catastrophes, Sleep and public policy: consensus report. Sleep 1988; 11: 100-109

35 Moscowitz H, Burns M. The effects of alcohol and valium, singly and in combination, upon driving-related skills performance. Proceedings of the $21^{\text {st }}$ Conference of the Association for Automobile Medicine. Vancouver, BC, 15-17. September 1977: 226-240

${ }^{36}$ Moscowitz H, Robison CD. The effect of low dosis of alcoholon driving related skills: a review of the evidence. Report No. DOT HS-807-280. Springfield Virginia National Technical Information Service; 121: 90-94

${ }^{37}$ Posner M, Rafal R. Cognitive theories of attention and the rehabilitation of attentional deficits. In: Meier M, Benton A, Diller L (eds.): Neuropsychological Rehabilitation. Edinburgh: 1987: 182 - 201

38 Posner MI, Petersen SE. The attention system of the human brain. Ann Rev Neurosciences 1990; 13: 25-42

${ }^{39}$ Randerath W, Gerdesmeyer C, Ströhlein G et al. Messung der Vigilanz mittels Fahrsimulator vor und nach nCPAP - Vergleich zweier Simulationsprogramme mit unterschiedlicher Ereignishäufigkeit. Somnologie $1997 ; 1: 110-114$

${ }^{40}$ Randerath W, Gerdesmeyer C, Siller C et al. A test for the determination of sustained attention in patients with obstructive sleep apnea syndrome. Respiration 2000; 67: 526-532

${ }^{41}$ Roehrs T, Zoricks F, Wittig R et al. Predictors of objective level of daytime sleepiness in patients with sleep-related breathing disorders. Chest 1989; 95: $1202-1206$

42 Rützel E. Aufmerksamkeit und Konzentration. Klinkhardt, Bad Heilbronn/Obb., 1982

${ }^{43}$ Walsleben JA. The measurement of daytime wakefulness. Chest 1992; 101: 890-891

${ }^{44}$ Weeß HG, Sauter C, Geißler P et al. Vigilanz, Einschlafneigung, Daueraufmerksamkeit, Müdigkeit, Schläfrigkeit - Diagnostische Instrumentearien zur Messung müdigkeits- und schläfrigkeitsbedingter Prozesse und deren Gütekriterien. Somnologie 2000; 4: 20-38 